\title{
REFINED ALMOST DOUBLE DERIVATIONS AND LIE *-DOUBLE DERIVATIONS
}

\author{
ISMAIL NIKOUFAR \\ Received 19 February, 2015
}

\begin{abstract}
In this paper, our approach allows to refine the results announced by Ebadian et al. [Results Math., 36 (2013), 409-423]. Namely, we reduce the distance between approximate and exact double derivations on Banach algebras and Lie $C^{*}$-algebras up to $\frac{1}{2^{n-1}}$ and $\frac{1}{2^{n-2}}$ for $n \geq 2$. Indeed, we give a correct utilization of fixed point theory in the sense of Diaz and Margolis [Bull. Amer. Math. Soc., 74 (1968), 305-309] concerning the stability of double derivations.
\end{abstract}

2010 Mathematics Subject Classification: 39B82; 39B52

Keywords: Hyers-Ulam stability, double derivation, $*$-double derivation, Lie $C^{*}$-algebra

\section{INTRODUCTION}

A classical question in the theory of functional equations is the following: when is it true that a mapping which approximately satisfies a functional equation $\xi$ must be somehow near to an exact solution of $\xi$ ?

In 1940, Ulam [7] gave a wide ranging talk and discussed a number of important unsolved problems. Among those was the question concerning the stability of group homomorphisms.

Let $\left(G_{1}, \cdot\right)$ be a group and let $\left(G_{2}, *\right)$ be a metric group with the metric $d(\cdot, \cdot)$. Given $\epsilon>0$, does there exist a $\delta>0$, such that if a mapping $h: G_{1} \longrightarrow G_{2}$ satisfies the inequality $d(h(x . y), h(x) * h(y))<\delta$ for all $x, y \in G_{1}$, then there exists $a$ homomorphism $H: G_{1} \longrightarrow G_{2}$ with $d(h(x), H(x))<\epsilon$ for all $x \in G_{1}$ ?

Generally, the concept of stability for a functional equation comes up when the functional equation is replaced by an inequality which acts as a perturbation of that equation. The case of approximately additive functions was solved by D. Hyers [5] under certain assumptions. In 1950, Hyers' Theorem was generalized by Aoki [1] for additive mappings and independently, in 1978, by Rassias [6] for linear mappings considering the Cauchy difference controlled by sum of powers of norms. For the history and various aspects of this theory we refer the reader to [3] and the references therein. Note that a functional equation $\zeta$ is stable if any function $g$ satisfying the equation $\zeta$ approximately is near to true solution of $\zeta$. 
Recently, Ebadian et al. [3] used the fixed point alternative method to establish the Hyers-Ulam stability of double derivations on Banach algebras and Lie $*$-double derivations on Lie $C^{*}$-algebras associated with the following additive functional equation

$$
\begin{aligned}
& \sum_{k=2}^{n}\left(\sum_{i_{1}=2}^{k} \sum_{i_{2}=i_{1}+1}^{k+1} \ldots \sum_{i_{n-k+1}=i_{n-k}+1}^{n}\right) f\left(\sum_{i=1, i \neq i_{1}, \ldots, i_{n-k+1}}^{n} x_{i}-\sum_{r=1}^{n-k+1} x_{i_{r}}\right) \\
& \quad+f\left(\sum_{i=1}^{n} x_{i}\right)=2^{n-1} f\left(x_{1}\right) .
\end{aligned}
$$

Throughout this paper following [3], we assume that $\mathcal{A}$ is a Banach algebra (Lie $C^{*}$ algebra). For given mapping $f: \mathcal{A} \rightarrow \mathcal{A}$, we define the difference operator $D_{\mu} f$ : $\mathcal{A}^{n} \rightarrow \mathcal{A}$ by

$$
\begin{aligned}
& D_{\mu} f\left(x_{1}, \ldots, x_{n}\right):= \\
& \quad \sum_{k=2}^{n}\left(\sum_{i_{1}=2}^{k} \sum_{i_{2}=i_{1}+1}^{k+1} \ldots \sum_{i_{n-k+1}=i_{n-k}+1}^{n}\right) f\left(\sum_{i=1, i \neq i_{1}, \ldots, i_{n-k+1}}^{n} \mu x_{i}-\sum_{r=1}^{n-k+1} \mu x_{i_{r}}\right) \\
& \quad+f\left(\sum_{i=1}^{n} \mu x_{i}\right)-2^{n-1} f\left(\mu x_{1}\right)
\end{aligned}
$$

for all $\mu \in\{\lambda \in \mathbb{C}:|\lambda|=1\}$.

In this paper, we improve main results of [3] and reduce the distance between approximate and exact double derivations on Banach algebras and Lie $C^{*}$-algebras up to $\frac{1}{2^{n-1}}$ and $\frac{1}{2^{n-2}}$ for $n \geq 2$.

In section 2, we discuss on main results of [3] and improve some theorems and corollaries including Theorem 2.3, 2.5 and Corollary 2.4, 2.6. In section 3, we also refine some results of [3] including Theorem 3.2, 3.4 and Corollary 3.3, 3.5. Indeed, we are going to weaken their assumptions and giving a correct utilization of fixed point theory in the sense of Diaz and Margolis [2].

\section{Almost Double DeRivation}

Throughout this section, we assume that $\mathcal{A}$ is a Banach algebra, $f(0)=g(0)=$ $h(0)=0$, and for given mappings $f, g, h: \mathcal{A} \longrightarrow \mathcal{A}$, we define

$$
C_{f, g, h}(a, b):=f(a b)-f(a) b-a f(b)-g(a) h(b)-h(a) g(b)
$$

for all $a, b \in \mathcal{A}$.

Definition 1. Let $\mathcal{A}$ be a Banach algebra and let $\delta, \varepsilon: \mathcal{A} \longrightarrow \mathcal{A}$ be $\mathbb{C}$-linear mappings. A $\mathbb{C}$-linear mapping $f: \mathcal{A} \longrightarrow \mathcal{A}$ is called a $(\delta, \varepsilon)$-double derivation if

$$
f(a b)=f(a) b+a f(b)+\delta(a) \varepsilon(b)+\varepsilon(a) \delta(b)
$$


for all $a, b \in \mathcal{A}$.

A fundamental result in fixed point theory is the following theorem. We recall the following theorem by Diaz and Margolis [2].

Theorem 1. Let $(X, d)$ be a complete generalized metric space and let $J: X \longrightarrow$ $X$ be a strictly contractive mapping with Lipschitz constant $0<L<1$. Then for each given element $x \in X$, either $d\left(J^{n} x, J^{n+1} x\right)=\infty$ for all $n \geq 0$, or there exists a natural number $n_{0}$ such that

(1) $d\left(J^{n} x, J^{n+1} x\right)<\infty$ for all $n \geq n_{0}$,

(2) the sequence $\left\{J^{n} x\right\}$ converges to a fixed point $y^{*}$ of $J$,

(3) $y^{*}$ is the unique fixed point of $J$ in the set $Y=\left\{y \in X: d\left(J^{n_{0}} x, y\right)<\infty\right\}$,

(4) $d\left(y, y^{*}\right) \leq \frac{1}{1-L} d(y, J y)$ for all $y \in Y$.

The following theorem is a refined version of [3, Theorem 2.3]:

Theorem 2. Let $f, g, h: \mathcal{A} \longrightarrow \mathcal{A}$ be mappings for which there exist functions $\varphi: \mathcal{A}^{n} \longrightarrow[0, \infty)$ and $\psi: \mathcal{A}^{2} \longrightarrow[0, \infty)$ such that

$$
\begin{gathered}
\lim _{m \rightarrow \infty} 2^{m} \varphi\left(\frac{x_{1}}{2^{m}}, \ldots, \frac{x_{n}}{2^{m}}\right)=0, \\
\lim _{m \rightarrow \infty} 4^{m} \psi\left(\frac{a}{2^{m}}, \frac{b}{2^{m}}\right)=0, \\
\left\|D_{\mu} f\left(x_{1}, \ldots, x_{n}\right)\right\| \leq \varphi\left(x_{1}, \ldots, x_{n}\right), \\
\left\|C_{f, g, h}(a, b)\right\| \leq \psi(a, b)
\end{gathered}
$$

for all $a, b, x_{1}, \ldots, x_{2} \in \mathcal{A}$ and all $\mu \in \mathbb{T}^{1}$. If there exists a constant $0<L<1$ such that $\varphi\left(x_{1}, \ldots, x_{n}\right) \leq \frac{L}{2} \varphi\left(2 x_{1}, \ldots, 2 x_{n}\right)$ for all $x_{1}, \ldots, x_{n} \in \mathcal{A}$, then there exist unique $\mathbb{C}$-linear mappings $d, \delta, \varepsilon: \mathcal{A} \rightarrow \mathcal{A}$ such that

$$
\begin{gathered}
\|f(x)-d(x)\| \leq \frac{L}{\alpha(1-L)} \varphi(x, x, 0, \ldots, 0), \\
\|g(x)-\delta(x)\| \leq \frac{L}{\alpha(1-L)} \varphi(x, x, 0, \ldots, 0), \\
\|h(x)-\varepsilon(x)\| \leq \frac{L}{\alpha(1-L)} \varphi(x, x, 0, \ldots, 0)
\end{gathered}
$$

for all $x \in \mathcal{A}$, where $\alpha=2^{n-1}$ and $n \geq 2$. Moreover, $d$ is a $(\delta, \varepsilon)$-double derivation on A.

Proof. Put $\mu=1, x_{1}=x_{2}=x$, and $x_{3}=x_{4}=\ldots=x_{n}=0$ in (2.3) to reach

$$
\left\|\frac{\alpha}{2} f(2 x)-\alpha f(x)\right\| \leq \varphi(x,, x, 0, \ldots, 0)
$$

for all $x \in \mathcal{A}$ and so

$$
\left\|2 f\left(\frac{x}{2}\right)-f(x)\right\| \leq \frac{2}{\alpha} \varphi\left(\frac{x}{2}, \frac{x}{2}, 0, \ldots, 0\right) \leq \frac{L}{\alpha} \varphi(x,, x, 0, \ldots, 0) .
$$


Define $F:=\{f: \mathcal{A} \longrightarrow \mathcal{A}\}$. The metric defined on $F$ by

$$
\rho(f, g):=\inf \{c \in[0, \infty]:\|f(x)-g(x)\| \leq c \varphi(x, x, 0, \ldots, 0), \forall x \in \mathcal{A}\} .
$$

is a generalized metric and $(F, \rho)$ is a generalized complete metric space. Consider the mapping $(J f)(x):=2 f\left(\frac{x}{2}\right)$ for all $f \in F$ and $x \in \mathcal{A}$. Use [4, Lemma 1.3] to see that $J$ is a strictly contractive mapping with the Lipschitz constant $L$. It follows from (2.8) that $\rho(J f, f) \leq \frac{L}{\alpha}$. Therefore according to Theorem 1 , the sequence $\left\{J^{m} f\right\}$ converges to a fixed point $d$ such that $d(x)=\lim _{m \rightarrow \infty} 2^{m} f\left(\frac{x}{2^{m}}\right)$ and $d(2 x)=2 d(x)$. Note that $d$ is the unique fixed point of $J$ and

$$
\rho(d, f) \leq \frac{1}{1-L} \rho(J f, f) \leq \frac{L}{\alpha(1-L)} .
$$

This means that inequality (2.5) holds for all $x \in \mathcal{A}$. The proof of the linearity of $d$ and also the rest of the proof is similar to that of [3, Theorem 2.3] and we omit it.

The importance of our result becomes clear when we take

$$
\varphi\left(x_{1}, \ldots, x_{n}\right)=\theta_{1} \sum_{i=1}^{n}\left\|x_{i}\right\|^{p}, \psi(a, b)=\theta_{2}\left(\|a\|^{q}+\|b\|^{q}\right) .
$$

In this situation, by choosing $L=2^{1-p}$, we can get strong and close approximations of the functions $f, g, h$ with linear mappings $d, \delta, \varepsilon$, where $d$ is a $(\delta, \varepsilon)$-double derivation on $\mathcal{A}$. Thus, we improve [3, Corollary 2.4] up to $\frac{1}{2^{n-1}}$ as follows:

Corollary 1. Let $p, q, \theta_{1}, \theta_{2}$ be non-negative real numbers with $p, q>1$. Suppose that $f, g, h: \mathcal{A} \longrightarrow \mathcal{A}$ are mappings such that

$$
\begin{aligned}
& \left\|D_{\mu} f\left(x_{1}, \ldots, x_{n}\right)\right\| \leq \theta_{1} \sum_{i=1}^{n}\left\|x_{i}\right\|^{p}, \\
& \left\|C_{f, g, h}(a, b)\right\| \leq \theta_{2}\left(\|a\|^{q}+\|b\|^{q}\right)
\end{aligned}
$$

for all $a, b, x_{1}, \ldots, x_{n} \in \mathcal{A}$ and all $\mu \in \mathbb{T}^{1}$. Then there exist unique $\mathbb{C}$-linear mappings $d, \delta, \varepsilon: \mathcal{A} \rightarrow \mathcal{A}$ such that

$$
\begin{aligned}
& \|f(x)-d(x)\| \leq \frac{2 \theta_{1}}{2^{n-1}\left(2^{p-1}-1\right)}\|x\|^{p}, \\
& \|g(x)-\delta(x)\| \leq \frac{2 \theta_{1}}{2^{n-1}\left(2^{p-1}-1\right)}\|x\|^{p}, \\
& \|h(x)-\varepsilon(x)\| \leq \frac{2 \theta_{1}}{2^{n-1}\left(2^{p-1}-1\right)}\|x\|^{p}
\end{aligned}
$$

for all $x \in \mathcal{A}$. Moreover, $d$ is a $(\delta, \varepsilon)$-double derivation on $\mathcal{A}$.

In the following theorem we give an improved version of [3, Theorem 2.5]: 
Theorem 3. Suppose that $f, g, h: \mathcal{A} \longrightarrow \mathcal{A}$ are mappings satisfying (2.3) and (2.4) for which there exist functions $\varphi: \mathscr{A}^{n} \longrightarrow[0, \infty)$ and $\psi: \mathcal{A}^{2} \longrightarrow[0, \infty)$ such that

$$
\begin{gathered}
\lim _{m \rightarrow \infty} \frac{1}{2^{m}} \varphi\left(2^{m} x_{1}, \ldots, 2^{m} x_{n}\right)=0, \\
\lim _{m \rightarrow \infty} \frac{1}{2^{m}} \psi\left(2^{m} a, 2^{m} b\right)=0
\end{gathered}
$$

for all $a, b, x_{1}, \ldots, x_{2} \in \mathcal{A}$. If there exists a constant $0<L<1$ such that $\varphi\left(x_{1}, \ldots, x_{n}\right) \leq$ $2 L \varphi\left(\frac{x_{1}}{2}, \ldots, \frac{x_{n}}{2}\right)$ for all $x_{1}, \ldots, x_{n} \in \mathcal{A}$, then there exist unique $\mathbb{C}$-linear mappings $d, \delta, \varepsilon: \mathcal{A} \rightarrow \mathcal{A}$ such that

$$
\begin{aligned}
\|f(x)-d(x)\| & \leq \frac{L}{\beta(1-L)} \varphi\left(\frac{x}{2}, \frac{x}{2}, 0, \ldots, 0\right), \\
\|g(x)-\delta(x)\| & \leq \frac{L}{\beta(1-L)} \varphi\left(\frac{x}{2}, \frac{x}{2}, 0, \ldots, 0\right), \\
\|h(x)-\varepsilon(x)\| & \leq \frac{L}{\beta(1-L)} \varphi\left(\frac{x}{2}, \frac{x}{2}, 0, \ldots, 0\right)
\end{aligned}
$$

for all $x \in \mathcal{A}$, where $\beta=\frac{\alpha}{2}$ and $n \geq 2$. Moreover, $d$ is a $(\delta, \varepsilon)$-double derivation on A.

Proof. It follows from (2.8) that

$$
\left\|\frac{1}{2} f(2 x)-f(x)\right\| \leq \frac{1}{\alpha} \varphi(x,, x, 0, \ldots, 0) \leq \frac{2 L}{\alpha} \varphi\left(\frac{x}{2}, \frac{x}{2}, 0, \ldots, 0\right)
$$

for all $x \in \mathcal{A}$. Consider the generalized complete metric $(F, \rho)$ with the generalized metric $\rho$ defined by

$$
\rho(f, g):=\inf \left\{c \in[0, \infty]:\|f(x)-g(x)\| \leq c \varphi\left(\frac{x}{2}, \frac{x}{2}, 0, \ldots, 0\right), \forall x \in \mathcal{A}\right\} .
$$

Define the mapping $(J f)(x):=\frac{1}{2} f(2 x)$ for all $f \in F$ and $x \in \mathcal{A}$. Apply [4, Lemma $1.2])$ to find that $J$ is a strictly contractive mapping with the Lipschitz constant $L$. It follows from (2.14) that $\rho(J f, f) \leq \frac{2 L}{\alpha}$. Applying Theorem 1, we get the sequence $\left\{J^{m} f\right\}$ converges to a unique fixed point $d$ of $J$ such that

$$
\rho(d, f) \leq \frac{1}{1-L} \rho(J f, f) \leq \frac{2 L}{\alpha(1-L)}=\frac{L}{\beta(1-L)},
$$

i. e., inequality (2.11) holds for all $x \in \mathcal{A}$. The rest of the proof is similar to that of [3, Theorem 2.3].

As we mentioned in Corollary 1, the importance of Theorem 3 becomes also clear when we put $L=2^{p-1}$ and

$$
\varphi\left(x_{1}, \ldots, x_{n}\right)=\theta_{1}+\theta_{2} \sum_{i=1}^{n}\left\|x_{i}\right\|^{p}, \psi(a, b)=\theta_{1}+\theta_{2}\left(\|a\|^{q}+\|b\|^{q}\right) .
$$


However, we can improve [3, Corollary 2.6] up to $\frac{1}{2^{n-2}}$ as follows:

Corollary 2. Let $p, q, \theta_{1}, \theta_{2}$ be non-negative real numbers with $p, q \in(0,1)$. Suppose that $f, g, h: \mathcal{A} \longrightarrow \mathcal{A}$ are mappings such that

$$
\begin{aligned}
& \left\|D_{\mu} f\left(x_{1}, \ldots, x_{n}\right)\right\| \leq \theta_{1}+\theta_{2} \sum_{i=1}^{n}\left\|x_{i}\right\|^{p}, \\
& \left\|C_{f, g, h}(a, b)\right\| \leq \theta_{1}+\theta_{2}\left(\|a\|^{q}+\|b\|^{q}\right)
\end{aligned}
$$

for all $a, b, x_{1}, \ldots, x_{n} \in \mathcal{A}$ and all $\mu \in \mathbb{T}^{1}$. Then there exist unique $\mathbb{C}$-linear mappings $d, \delta, \varepsilon: \mathcal{A} \rightarrow \mathcal{A}$ such that

$$
\begin{gathered}
\|f(x)-d(x)\| \leq \frac{1}{2^{n-2}}\left(\frac{\theta_{1}}{2^{1-p}-1}+\frac{\theta_{2}}{1-2^{p-1}}\|x\|^{p}\right), \\
\|g(x)-\delta(x)\| \leq \frac{1}{2^{n-2}}\left(\frac{\theta_{1}}{2^{1-p}-1}+\frac{\theta_{2}}{1-2^{p-1}}\|x\|^{p}\right), \\
\|h(x)-\varepsilon(x)\| \leq \frac{1}{2^{n-2}}\left(\frac{\theta_{1}}{2^{1-p}-1}+\frac{\theta_{2}}{1-2^{p-1}}\|x\|^{p}\right)
\end{gathered}
$$

for all $x \in \mathcal{A}$. Moreover, $d$ is a $(\delta, \varepsilon)$-double derivation on $\mathcal{A}$.

\section{Almost LIE $*$-DOUble DERIVATION}

A unital $C^{*}$-algebra $\mathcal{A}$, endowed with the Lie product $[x, y]=x y-y x$ on $\mathcal{A}$, is called a Lie $C^{*}$-algebra. In this section, we assume that $\mathcal{A}$ is a Lie $C^{*}$-algebra and $U(\mathcal{A})=\left\{u \in \mathcal{A}: u u^{*}=u^{*} u=e\right\}$. For given mappings $f, g, h: \mathcal{A} \longrightarrow \mathcal{A}$, we let $f(0)=g(0)=h(0)=0$ and define

$$
J_{f, g, h}(a, b):=f([a, b])-[f(a), b]-[a, f(b)]-[g(a), h(b)]-[h(a), g(b)]
$$

for all $a, b \in \mathcal{A}$.

Definition 2. Let $\mathcal{A}$ be a Lie $C^{*}$-algebra and let $\delta, \varepsilon: \mathcal{A} \longrightarrow \mathcal{A}$ be $\mathbb{C}$-linear mappings. A $\mathbb{C}$-linear mapping $f: \mathcal{A} \longrightarrow \mathcal{A}$ is called a Lie $(\delta, \varepsilon)$-double derivation if

$$
f([a, b])=[f(a), b]+[a, f(b)]+[\delta(a), \varepsilon(b)]+[\varepsilon(a), \delta(b)]
$$

for all $a, b \in \mathcal{A}$.

The presented results in this section are refinements of [3, Theorem 3.2, 3.4] and [3, Corollary 3.3, 3.5]:

Theorem 4. Let $f, g, h: \mathcal{A} \longrightarrow \mathcal{A}$ be mappings for which there exist functions $\varphi: \mathcal{A}^{n} \longrightarrow[0, \infty)$ and $\psi: \mathcal{A}^{2} \longrightarrow[0, \infty)$ such that

$$
\begin{gathered}
\lim _{m \rightarrow \infty} 2^{m} \varphi\left(\frac{x_{1}}{2^{m}}, \ldots, \frac{x_{n}}{2^{m}}\right)=0, \\
\lim _{m \rightarrow \infty} 4^{m} \psi\left(\frac{a}{2^{m}}, \frac{b}{2^{m}}\right)=0, \\
\left\|D_{\mu} f\left(x_{1}, \ldots, x_{n}\right)\right\| \leq \varphi\left(x_{1}, \ldots, x_{n}\right),
\end{gathered}
$$




$$
\begin{gathered}
\left\|J_{f, g, h}(a, b)\right\| \leq \psi(a, b) \\
\max \left\{f\left(\frac{u^{*}}{2^{k}}\right)-f\left(\frac{u}{2^{k}}\right)^{*}, g\left(\frac{u^{*}}{2^{k}}\right)-g\left(\frac{u}{2^{k}}\right)^{*}, h\left(\frac{u^{*}}{2^{k}}\right)-h\left(\frac{u}{2^{k}}\right)^{*}\right\} \leq \varphi\left(\frac{u}{2^{k}}, \ldots, \frac{u}{2^{k}}\right)
\end{gathered}
$$

for all $a, b, x_{1}, \ldots, x_{2} \in \mathcal{A}, k=0,1,2, \ldots, u \in U(A)$, and $\mu \in \mathbb{T}^{1}$. If there exists $a$ constant $0<L<1$ such that $\varphi\left(x_{1}, \ldots, x_{n}\right) \leq \frac{L}{2} \varphi\left(2 x_{1}, \ldots, 2 x_{n}\right)$ for all $x_{1}, \ldots, x_{n} \in \mathcal{A}$, then there exist unique $\mathbb{C}$-linear mappings $d, \delta, \varepsilon: \mathcal{A} \rightarrow \mathcal{A}$ such that

$$
\max \{\|f(x)-d(x)\|,\|g(x)-\delta(x)\|,\|h(x)-\varepsilon(x)\|\} \leq \frac{L}{\alpha(1-L)} \varphi(x, x, 0, \ldots, 0)
$$

for all $x \in \mathcal{A}$. Moreover, $d$ is a Lie $*-(\delta, \varepsilon)$-double derivation on $\mathcal{A}$.

Proof. Using the same methods as in the proof of [3, Theorem 2.3, 3.2], we can obtain the desired results.

Corollary 3. Let $p, q, \theta_{1}, \theta_{2}$ be non-negative real numbers with $p, q>1$. Suppose that $f, g, h: \mathcal{A} \longrightarrow \mathcal{A}$ are mappings such that

$$
\begin{gathered}
\left\|D_{\mu} f\left(x_{1}, \ldots, x_{n}\right)\right\| \leq \theta_{1} \sum_{i=1}^{n}\left\|x_{i}\right\|^{p}, \\
\left\|J_{f, g, h}(u, b)\right\| \leq \theta_{2}\left(1+\|b\|^{q}\right) \\
\max \left\{f\left(\frac{u^{*}}{2^{k}}\right)-f\left(\frac{u}{2^{k}}\right)^{*}, g\left(\frac{u^{*}}{2^{k}}\right)-g\left(\frac{u}{2^{k}}\right)^{*}, h\left(\frac{u^{*}}{2^{k}}\right)-h\left(\frac{u}{2^{k}}\right)^{*}\right\} \leq \frac{\theta_{1}+\theta_{2}}{2^{k p}}
\end{gathered}
$$

for all $a, b, x_{1}, \ldots, x_{n} \in \mathcal{A}, k=0,1,2, \ldots, u \in U(A)$, and $\mu \in \mathbb{T}^{1}$. There exist unique $\mathbb{C}$-linear mappings $d, \delta, \varepsilon: \mathcal{A} \rightarrow \mathcal{A}$ such that

$$
\max \{\|f(x)-d(x)\|,\|g(x)-\delta(x)\|,\|h(x)-\varepsilon(x)\|\} \leq \frac{2 \theta_{1}}{2^{n-1}\left(2^{p-1}-1\right)}\|x\|^{p}
$$

for all $x \in \mathcal{A}$. Moreover, $d$ is a $(\delta, \varepsilon)$-double derivation on $\mathcal{A}$.

Proof. The results follows from above theorem by taking $L=2^{1-p}$ and

$$
\varphi\left(x_{1}, \ldots, x_{n}\right)=\theta_{1} \sum_{i=1}^{n}\left\|x_{i}\right\|^{p}, \psi(a, b)=\theta_{2}\left(1+\|b\|^{q}\right) .
$$

Theorem 5. Suppose that $f, g, h: \mathcal{A} \longrightarrow \mathcal{A}$ are mappings satisfying (3.3) and (3.4) for which there exist functions $\varphi: \mathcal{A}^{n} \longrightarrow[0, \infty)$ and $\psi: \mathcal{A}^{2} \longrightarrow[0, \infty)$ such that

$$
\begin{gathered}
\lim _{m \rightarrow \infty} \frac{1}{2^{m}} \varphi\left(2^{m} x_{1}, \ldots, 2^{m} x_{n}\right)=0, \\
\lim _{m \rightarrow \infty} \frac{1}{4^{m}} \psi\left(2^{m} a, 2^{m} b\right)=0 \\
\max \left\{f\left(2^{k} u^{*}\right)-f\left(2^{k} u\right)^{*}, g\left(2^{k} u^{*}\right)-g\left(2^{k} u\right)^{*}, h\left(2^{k} u^{*}\right)-h\left(2^{k} u\right)^{*}\right\} \leq \varphi\left(2^{k} u, \ldots 2^{k} u\right)
\end{gathered}
$$


for all $a, b, x_{1}, \ldots, x_{2} \in \mathcal{A}, k=0,1,2, \ldots, u \in U(A)$, and $\mu \in \mathbb{T}^{1}$. If there exists $a$ constant $0<L<1$ such that $\varphi\left(x_{1}, \ldots, x_{n}\right) \leq 2 L \varphi\left(\frac{x_{1}}{2}, \ldots, \frac{x_{n}}{2}\right)$ for all $x_{1}, \ldots, x_{n} \in \mathcal{A}$, then there exist unique $\mathbb{C}$-linear mappings $d, \delta, \varepsilon: \mathcal{A} \rightarrow \mathcal{A}$ such that

$$
\max \{\|f(x)-d(x)\|,\|g(x)-\delta(x)\|,\|h(x)-\varepsilon(x)\|\} \leq \frac{L}{\beta(1-L)} \varphi\left(\frac{x}{2}, \frac{x}{2}, 0, \ldots, 0\right)
$$

for all $x \in \mathcal{A}$, where $\beta=\frac{\alpha}{2}$ and $n \geq 2$. Moreover, $d$ is a Lie $*-(\delta, \varepsilon)$-double derivation on $\mathcal{A}$.

Proof. The proof is similar to that of [3, Theorem 2.5, 3.2].

Corollary 4. Let $p, q, \theta_{1}, \theta_{2}$ be non-negative real numbers with $p, q \in(0,1)$. Suppose that $f, g, h: \mathcal{A} \longrightarrow \mathcal{A}$ are mappings such that

$$
\begin{gathered}
\left\|D_{\mu} f\left(x_{1}, \ldots, x_{n}\right)\right\| \leq \theta_{1}+\theta_{2} \sum_{i=1}^{n}\left\|x_{i}\right\|^{p}, \\
\left\|J_{f, g, h}(u, b)\right\| \leq \theta_{1}+\theta_{2}\left(1+\|b\|^{q}\right) \\
\max \left\{f\left(2^{k} u^{*}\right)-f\left(2^{k} u\right)^{*}, g\left(2^{k} u^{*}\right)-g\left(2^{k} u\right)^{*}, h\left(2^{k} u^{*}\right)-h\left(2^{k} u\right)^{*}\right\} \leq \frac{\theta_{1}+\theta_{2}}{2^{k p}}
\end{gathered}
$$

for all $a, b, x_{1}, \ldots, x_{n} \in \mathcal{A}, k=0,1,2, \ldots, u \in U(A)$, and $\mu \in \mathbb{T}^{1}$. Then there exist unique $\mathbb{C}$-linear mappings $d, \delta, \varepsilon: \mathcal{A} \rightarrow \mathcal{A}$ such that

$$
\begin{gathered}
\max \{\|f(x)-d(x)\|,\|g(x)-\delta(x)\|,\|h(x)-\varepsilon(x)\|\} \\
\leq \frac{1}{2^{n-2}}\left(\frac{\theta_{1}}{2^{1-p}-1}+\frac{\theta_{2}}{1-2^{p-1}}\|x\|^{p}\right)
\end{gathered}
$$

for all $x \in \mathcal{A}$. Moreover, $d$ is a Lie $*-(\delta, \varepsilon)$-double derivation on $\mathcal{A}$.

Proof. Apply above theorem by putting $L=2^{p-1}$ and

$$
\varphi\left(x_{1}, \ldots, x_{n}\right)=\theta_{1}+\theta_{2} \sum_{i=1}^{n}\left\|x_{i}\right\|^{p}, \psi(a, b)=\theta_{1}+\theta_{2}\left(1+\|b\|^{q}\right) .
$$

\section{CONCLUSION}

Our results can give the results proved by Ebadian et al. [3]. For instance, under the hypotheses of Theorem 2 we can conclude [3, Theorem 2.3], but not vice versa. In other words, if there exists a constant $0<L<1$ such that $\varphi\left(x_{1}, \ldots, x_{n}\right) \leq$ $\frac{L}{2} \varphi\left(2 x_{1}, \ldots, 2 x_{n}\right)$ for all $x_{1}, \ldots, x_{n} \in \mathcal{A}$, then $\varphi\left(x_{1}, \ldots, x_{n}\right) \leq \frac{\alpha}{2} L \varphi\left(2 x_{1}, \ldots, 2 x_{n}\right)$, i.e, all of the hypotheses of [3, Theorem 2.3] hold. On the other hand, Theorem 2 says that there exist unique $\mathbb{C}$-linear mappings $d, \delta, \varepsilon: \mathcal{A} \rightarrow \mathcal{A}$ such that

$$
\|f(x)-d(x)\| \leq \frac{L}{\alpha(1-L)} \varphi(x, x, 0, \ldots, 0),
$$




$$
\begin{array}{r}
\|g(x)-\delta(x)\| \leq \frac{L}{\alpha(1-L)} \varphi(x, x, 0, \ldots, 0), \\
\|h(x)-\varepsilon(x)\| \leq \frac{L}{\alpha(1-L)} \varphi(x, x, 0, \ldots, 0)
\end{array}
$$

for all $x \in \mathcal{A}$, where $\alpha=2^{n-1}$ and $n \geq 2$. Since $\frac{L}{\alpha(1-L)} \leq \frac{L}{1-L}$, we have

$$
\begin{aligned}
\|f(x)-d(x)\| & \leq \frac{L}{1-L} \varphi(x, x, 0, \ldots, 0), \\
\|g(x)-\delta(x)\| & \leq \frac{L}{1-L} \varphi(x, x, 0, \ldots, 0), \\
\|h(x)-\varepsilon(x)\| & \leq \frac{L}{1-L} \varphi(x, x, 0, \ldots, 0),
\end{aligned}
$$

which coincide with the results of [3, Theorem 2.3]. The same arguments can be applied for Theorem 2.5, 3.2, 3.4 and Corollary 2.4, 2.6, 3.3, 3.5 of [3].

\section{REFERENCES}

[1] T. Aoki, "On the stability of the linear transformation in banach spaces," J. Math. Soc. Japan., vol. 2, pp. 64-66, 1950, doi: 10.2969/jmsj/00210064.

[2] J. B. Diaz and B. Margolis, "A fixed point theorem of the alternative for contractions on a generalized complete metric space," Bull. Amer. Math. Soc., vol. 74, pp. 305-309, 1968.

[3] A. Ebadian and N. Ghobadipour, "A fixed point approach to almost double derivations and lie *double derivations," Results Math., vol. 36, pp. 409-423, 2013, doi: 10.1007/s00025-011-0205-y.

[4] A. Ebadian, I. Nikoufar, T. M. Rassias, and N. Ghobadipour, "Stability of generalized derivations on hilbert $\mathrm{c}^{*}$-modules associated to a pexiderized cauchy-jensen type functional equation," Acta Math. Sci., vol. 32, no. 3, pp. 1226-1238, 2012, doi: 10.1016/S0252-9602(12)60094-0.

[5] D. H. Hyers, "On the stability of the linear functional equation," Proc. Natl. Acad. Sci., vol. 27, pp. 222-224, 1941.

[6] T. M. Rassias, "On the stability of the linear mapping in banach spaces," Proc. Amer. Math. Soc., vol. 72, pp. 297-300, 1978, doi: 10.1090/S0002-9939-1978-0507327-1.

[7] S. M. Ulam, Problems in Modern Mathematics. New York: Science Editions, Wiley, 1960.

\section{Author's address}

\section{Ismail Nikoufar}

Department of Mathematics, Payame Noor University, P.O. BOX 19395-3697 Tehran, Iran

E-mail address: nikoufarepnu.ac.ir 